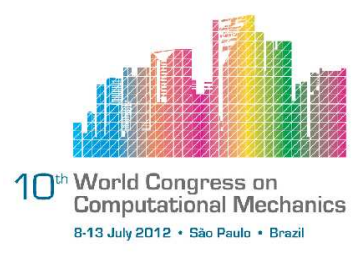

\title{
DESIGN OF COMPLIANT MECHANISMS WITH A SEQUENTIAL ELEMENT REJECTION AND ADMISSION METHOD
}

\author{
C. Alonso ${ }^{1}$, R. Ansola ${ }^{1}$, O.M. Querin ${ }^{2}$, J. Canales ${ }^{1}$ \\ ${ }^{1}$ Dept. of Mechanical Engineering, University of The Basque Country, Bilbao, Spain \\ (calonso015@ikasle.ehu.es)
}

${ }^{2}$ School of Mechanical Engineering, University of Leeds, Leeds, United Kingdom

\begin{abstract}
A Sequential Element Rejection and Admission (SERA) method to design compliant mechanisms with topology optimization techniques is presented in this work. This procedure, successfully applied to structural optimization problems, allows material to flow between two different material models: 'real' and 'virtual'. This bi-directional method works with two separate criterions for the rejection and admission of elements to efficiently achieve the optimum design. Three benchmark problems are presented here to demonstrate the validity of the proposed method.
\end{abstract}

Keywords: Optimization, topology, compliant mechanisms, SERA method

\section{INTRODUCTION}

A compliant mechanism is one that gains some or all of its mobility from the flexibility of its components. As a result, compliant mechanisms may be built from fewer parts, cutting the need for assembly procedures to a minimum. Other advantages are that they have less wear, friction or backlash and, as a result, no need for lubrication [1] [2].

Initially accomplished by trial and error, the idea of introducing more systematic procedures to the design of compliant mechanisms captured the mind of researchers [3]. Two different design approaches were considered: 1) Lumped and 2) distributed compliant mechanisms. In the first approach [4][5], rigid body mechanisms were converted into partially compliant mechanisms composed of small flexible pivots and rigid links. In the second approach, distributed compliant mechanisms were obtained with the use of topology optimization techniques. Optimum designs were automatically obtained for prescribed design domains, boundary conditions and functional specifications.

The pioneered topology optimization method used to design compliant mechanisms was the homogenization method [6]. However, the most widely used topology optimization method for compliant mechanisms has become SIMP [7]. In this approach, material properties were considered constant within each element and the element densities were the design variables. The effective property of each element consisted of its density raised to a power and 
multiplied to the material properties of the solid material. The SIMP method was applied to a variety of compliant mechanism design problems [8][9][10].

A number of heuristic or intuition based methods were also applied to the design of compliant mechanisms since the beginning of the 2000s. These were, among others: Genetic Algorithms [11], Level Set Methods [12] and the Evolutionary Structural Optimization (ESO) method [13]. The first two methods were also extended to cover further applications such as path generation mechanisms [14][15], design of multiphysics actuators [8][16] and non linear analysis [17][18][19].

The third of the methods, ESO, was not implemented further due to two issues: 1) The unidirectional nature of the procedure; and 2) a convoluted objective function which included the output stiffness, but did not allow for the control of the ratio between the input vs. output stiffness. The method proposed by Ansola et al. [13] used the additive version of the method, AESO [20]. The method worked by starting from an empty design domain (most compliant mechanism) with material gradually added until the target volume was achieved.

The aim of this paper is to present a method that overcomes the problems of the ESO methods to the design of compliant mechanisms. For this purpose, a Sequential Element Rejection and Additional (SERA) method [21] is proposed, that adds and removes material from the design domain. The problem is defined as the maximization of the Mutual Potential Energy (MPE) and with the ratio between the input and output stiffness controlled using a spring model [6]. A classical filtering technique [22] is used to avoid the formation of checkerboard patterns, giving the method the necessarily mesh-independency. Different benchmark problems are used to demonstrate the validity of the proposed method. In each example, only one specific problem parameter is varied to prove that the method is not sensitive to the selection of parameters in order to obtain an optimized topology, making SERA a robust method to design compliant mechanisms.

\section{PROBLEM FORMULATION AND SENSITIVITY ANALYSIS}

Figure 1a shows a compliant mechanism domain $\Omega$. It is subjected to a force $F_{\text {in }}$ at the input port $\mathrm{P}_{\text {in }}$ and is supposed to produce an output displacement $\Delta_{\text {out }}$ at the output port, $\mathrm{P}_{\text {out }}$.

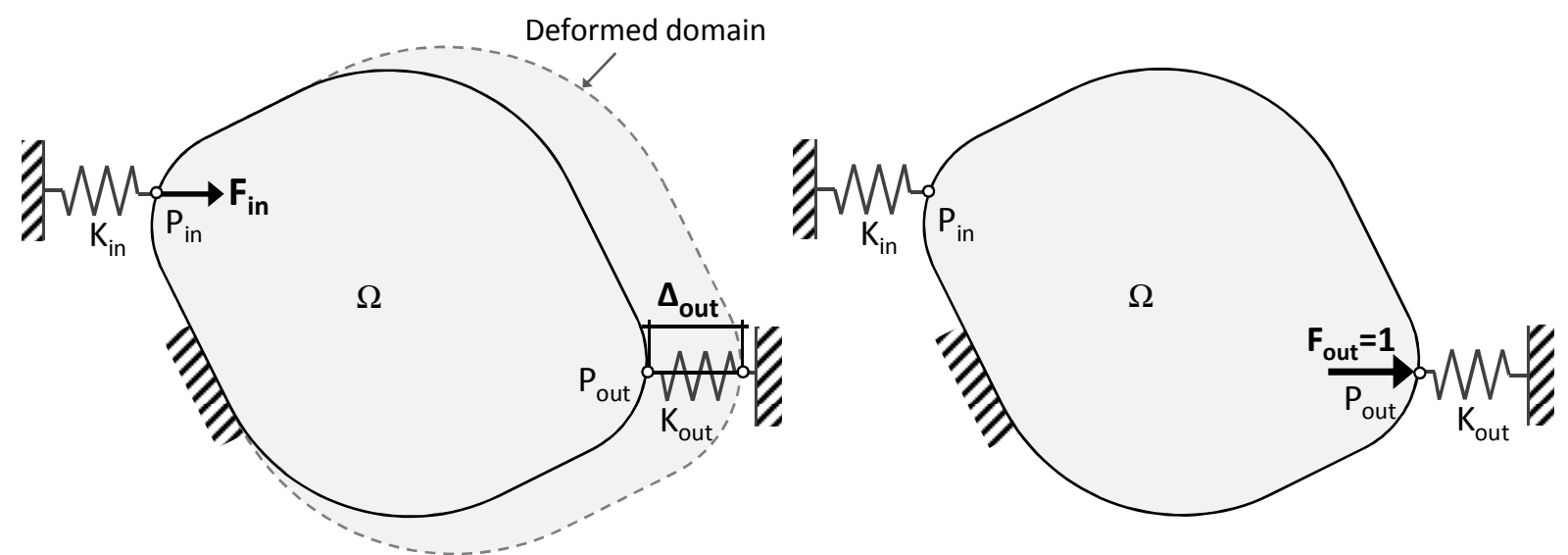

Figure 1. a) Problem definition of a compliant mechanism; b) Case 2: Pseudo-Force 
The goal of topology optimization for compliant mechanisms is to obtain the optimum design that converts an input work to a displacement or force in a predefined output direction. The mathematical formulation of this work is expressed as the maximization of the Mutual Potential Energy (MPE) (1) subjected to a target volume, V* (2).

$\max$ MPE

$$
\text { s.t. } \quad \sum_{e=1}^{N} \rho_{e} \leq V^{*}, \quad \rho_{e}=\left\{\rho_{\text {min }}, 1\right\}, e=1, \ldots, N
$$

where: $\rho_{\mathrm{e}}$ is the element density, $\mathrm{N}$ is the number of finite elements, and $\rho_{\min }$ is the minimum density considered. A typical value of $\rho_{\min }$ is $10^{-4}$.

The MPE (3) was defined as the deformation at a prescribed output point in a specified direction [23]. To obtain the MPE, two load cases need to be solved: 1) The Input Force Case, where the input force $\mathrm{F}_{\text {in }}$ is applied at the input port, $\mathrm{P}_{\mathrm{in}}$, named with the subscript 1 in equations $(3,4)$, Figure 1a; and 2) the Pseudo-Force Case, where a unit force is applied at the output point, $\mathrm{P}_{\text {out }}$ in the direction of the desired displacement, named with the subscript 2 in equations $(3,5)$, Figure $1 b$.

$$
\begin{aligned}
& M P E=\mathbf{U}_{2}{ }^{T} \cdot \mathbf{K} \cdot \mathbf{U}_{1} \\
& \mathbf{K} \cdot \mathbf{U}_{1}=\mathbf{F}_{1} \\
& \mathbf{K} \cdot \mathbf{U}_{2}=\mathbf{F}_{2}
\end{aligned}
$$

where: $\mathbf{K}$ is the global stiffness matrix of the structure; $\mathbf{F}_{1}$ is the nodal force vector containing the input force, $\mathrm{F}_{\text {in }} ; \mathbf{F}_{2}$ is the nodal force vector containing the unit output force; and $\mathbf{U}_{1}, \mathbf{U}_{2}$ are the displacement fields due to each load case.

The spring model of Figure 1a is used in this work to define the stiffness ratio between the input and output ports. The artificial input spring $\mathrm{k}_{\text {in }}$ together with a spring force $\mathrm{F}_{\text {in }}$ simulates the input work of the actuator. The resistance to the output displacement is modelled with a spring of stiffness $\mathrm{k}_{\text {out }}$. This allows the displacement amplification to be controlled by specifying different values of the output spring.

As part of the optimization process, a sensitivity analysis is carried out to provide information on how sensitive the MPE is to small changes in the design variables. This sensitivity number in each element (6) determines which elements are to be removed or added so that the objective function is maximized (see [13] for a complete explanation of the sensitivity analysis).

$$
\alpha_{e}=-\mathbf{U}_{1 e}^{T} \cdot \mathbf{K}_{e} \cdot \mathbf{U}_{2 e}
$$

where: $\mathbf{U}_{1 e}$ is the displacement vector of element $e$ due to load case $1 ; \mathbf{U}_{2 e}$ is the displacement vector of element $e$ due to load case 2 ; and $\mathbf{K}_{e}$ is the elemental stiffness matrix. 


\section{SERA FOR COMPLIANT MECHANISMS}

The Sequential Element Rejection and Admission (SERA) method is a bi-directional method that considers two separate material models: 1) 'Real' material and 2) a 'Virtual' material with negligible stiffness [21]. Two separate criterions of rejection and admission allow material to be redistributed from 'virtual' to 'real' and vice versa. The final topology is made of all the 'real' material present at the end of the optimization.

To apply SERA for compliant mechanisms, the concept of two material models and separate criterion is maintained and the driving criterion is necessarily adapted. Here a sensitivity analysis is performed and the resulting elemental sensitivity values are the ones that define the elements rejection and admission criteria.

The twelve steps that drive the SERA method for compliant mechanisms are given below, and can be seen in the flow chart of Figure 2.

1) Define the design problem. The maximum design domain must be defined and meshed with finite elements. All boundary constraints, loads and the target volume, $V^{*}$ must also be specified.

2) Assign 'real' and 'virtual' material properties to the initial design domain. Material present in the domain is assigned 'real' material properties and material not present is assigned 'virtual' material properties.

3) Calculate the target volumes to be added and removed in the $i^{\text {th }}$ iteration, $\Delta V_{\text {remove }}(i)$ and $\Delta V_{\text {add }}(i)$, (see section 3.1).

4) Carry out the Finite Element Analysis for the two load cases to produce the displacement vectors $\mathbf{U}_{1}$ and $\mathbf{U}_{2}$. The elemental and global stiffness matrixes, $\mathbf{K}_{\mathrm{e}}$ and $\mathbf{K}$, are also calculated as part of the FEA.

5) Calculate the elemental sensitivity numbers, $\alpha_{e},(6)$.

6) Apply a mesh independency filter to the sensitivity numbers.

7) Separate the sensitivity numbers in different lists for values related to the 'real' and 'virtual' materials, $\alpha_{\text {real }}$ and $\alpha_{\text {virtual }}$.

8) Define the threshold values for real and virtual material, $\alpha_{\text {real }}^{\text {th }}$ and $\alpha_{\text {virtual }}^{\text {th }}$. These values are the corresponding sensitivity values that remove or add the equivalent volumes, $\Delta V_{\text {remove }}(i)$ and $\Delta V_{\text {add }}(i)$.

9) Remove and add elements. For 'real' material, remove elements with lower $\alpha_{\text {real }}$; and for 'virtual' material, add elements with higher $\alpha_{\text {virtual }}$.

10) Calculate the volume of the 'real' material in the domain.

11) Calculate the convergence criterion, $\varepsilon_{i}(7)$. The convergence criterion is defined as the change in the objective function in the last 10 iterations. This implies that the process will have a minimum of 10 iterations as the convergence criterion is not applied until that number of iteration is reached. 


$$
\varepsilon_{i}=\frac{\left|\sum_{i-9}^{i-5} M P E_{i}-\sum_{i-4}^{i} M P E_{i}\right|}{\sum_{i-4}^{i} M P E_{i}}
$$

12) Repeat steps (3) through (11) until the objective volume is reached and the optimization converges. The final topology is represented by the 'real' material in the design domain.

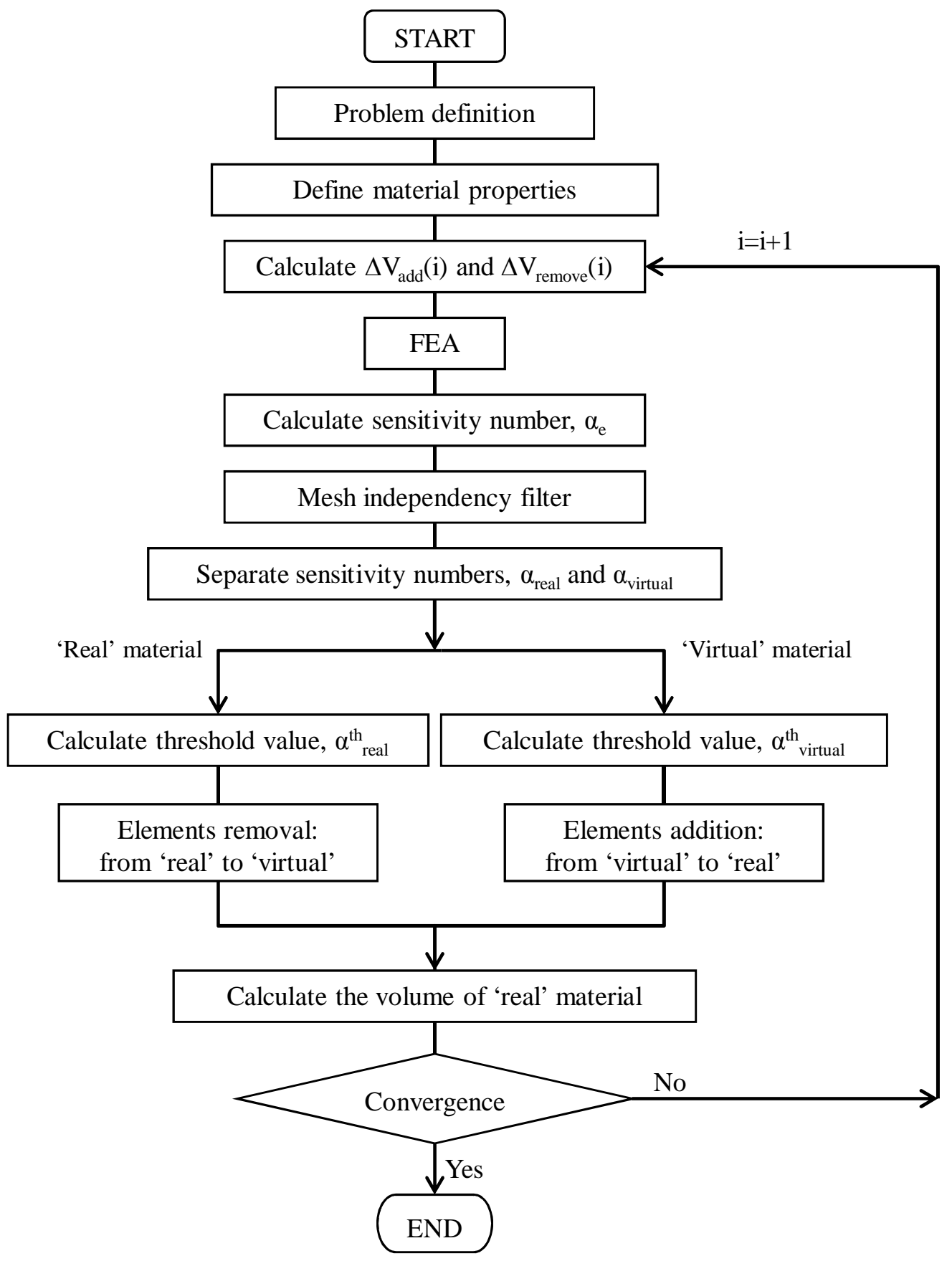

Figure 2. Flow chart of the SERA method for compliant mechanisms 


\subsection{Calculation of $\Delta V_{\text {remove }}(i)$ and $\Delta V_{\text {add }}(i)$}

The optimization process has two stages: $1^{\text {st }}$, when the process mainly adds (or removes) material until the target volume is reached; and $2^{\text {nd }}$, when the same amount of material is added and removed until convergence is achieved.

In the first stage, the target volume in each iteration is a function of the iteration number and is obtained differently depending if the initial design domain is full or void. If the process starts from a void design domain, the objective volume in the $i^{\text {th }}$ iteration, $V(i)$, is calculated using (8), and if it starts with a full design domain, $V(i)$ is obtained using equation (9).

For both case, the difference between the target volume at the present iteration, $V(i)$ and the previous one, $V(i-1)$, is the amount of material that has to be relocated, $\Delta V(i),(10)$.

$$
\begin{aligned}
& V(i)=\min \left((V(i-1)+P R), V^{*}\right) \\
& V(i)=\max \left(\left(V(i-1) \cdot(1-P R), V^{*}\right)\right. \\
& \Delta V(i)=|V(i)-V(i-1)|
\end{aligned}
$$

where: $P R$ is the Progress Ratio, defined by the user. Typical values of $P R$ are 0.01 0.05 and it defines the speed of achieving the target volume, $V^{*}$.

The general volume variation $\Delta V(i)$ needs to be spitted in separate values for the target volume to be added, $\Delta V_{\text {add }}(i)$ and removed, $\Delta V_{\text {remove }}(i)$ as SERA uses separate criteria for each of those processes.

These two values are also different if the initial design domain is full or void. For an initial void design domain, a bigger amount of material is added (11) to allow some elements to be then removed (12).

$$
\begin{aligned}
& \Delta V_{\text {add }}(i)=S R \cdot \Delta V(i) \\
& \Delta V_{\text {removed }}(i)=\Delta V(i) \cdot(S R-1)
\end{aligned}
$$

where: $S R$ is the Smoothing Ratio. Typical values of $S R$ are $1.2-1.4$ so that an additional amount of material is added in each iteration and that same amount removed.

The reverse is done for a full initial design domain, (13) and (14).

$$
\begin{aligned}
& \Delta V_{\text {remove }}(i)=S R \cdot \Delta V(i) \\
& \Delta V_{\text {add }}(i)=\Delta V(i) \cdot(S R-1)
\end{aligned}
$$

With this approach, a bi-directional procedure is used since the beginning of the process as material is both added and removed since the beginning and not only when the target volume has been reached.

In the second stage of the process, when the volume has reached the target value, the same amount of material is removed and added so that the topology can be optimized and the outline better defined until it reaches convergence. A typical value at this stage is the following:

$$
\Delta V_{\text {add }}(i)=\Delta V_{\text {remove }}(i)=0.1 \cdot P R \cdot N
$$

where: $N$ is the number of elements in the design domain. 


\section{EXAMPLES}

Three benchmark problems are presented in this section: 1) An inverter mechanism 2) a crunching mechanism and 3) a gripper mechanism. For each example, a problem parameter is varied to prove the robustness of the method in achieving an optimized topology regardless of the problem parameters used.

The material properties used in all examples are the same. The Young's modulus is $\mathrm{E}=200 \mathrm{GPa}$ and the Poisson's ratio $v=0.3$. The density of the virtual material is $\rho_{\mathrm{min}}=10^{-4}$, which is equivalent to $0.01 \%$ of the stiffness of the real material.

\subsection{Inverter mechanism with different mesh densities}

The design domain for an inverter mechanism is shown in Figure 3. It is a square of size 200x200mm subdivided using square four node finite elements.

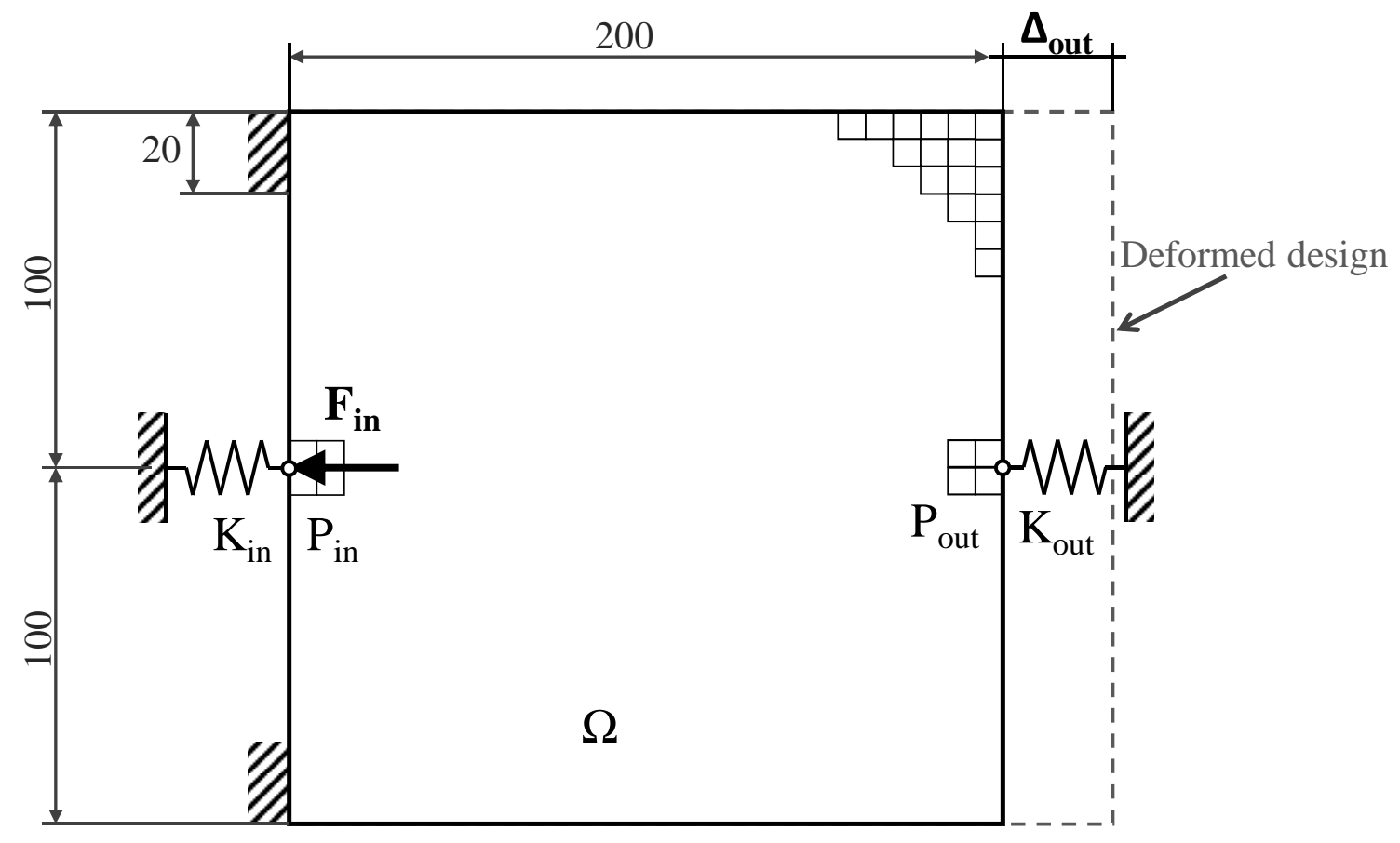

Figure 3. Inverter mechanism (all dimensions are in $\mathrm{mm}$ )

In this section, the parameter varied is the mesh density to demonstrate the mesh independency of the method. A robust method shall achieve the same optimum topology regardless of the number of finite elements in which the design domain is subdivided.

The 3 mesh densities considered are: 1) Coarse, consisting of 400 10x10mm finite elements; 2) Medium, consisting of $16005 \times 5 \mathrm{~mm}$ finite elements; 3) Fine, consisting of $10,0002 \times 2 \mathrm{~mm}$ finite elements. In all cases, the same input force $\mathrm{F}_{\mathrm{in}}=1 \mathrm{~N}$ is applied and a stiffness ratio of $\mathrm{k}_{\text {out }} / \mathrm{k}_{\mathrm{in}}=1$ is defined. The target volume, $V^{*}$ is $40 \%$ of the initial design domain and the filter radius used in all cases is $r_{\min }=6 \mathrm{~mm}$. Results for the three cases are shown in Figure 4. 


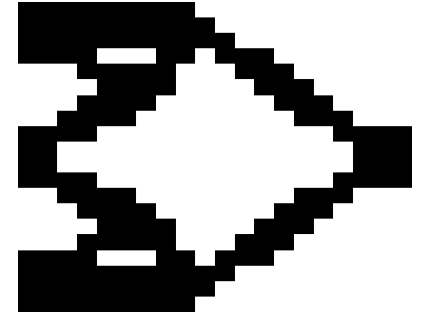

a

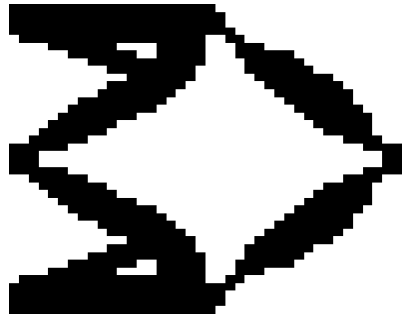

b

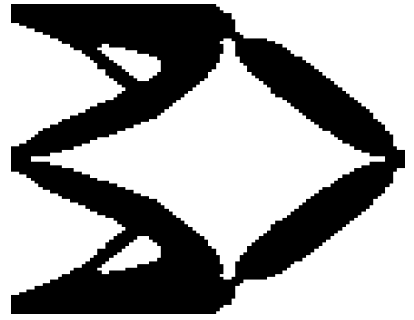

$\mathrm{C}$

Figure 4. Inverter mechanism designs for the different mesh densities: a) Coarse; b) Medium;

c) Fine

As it can be observed, the resulting topologies are the same for the three mesh densities and comparable with the optimum topologies obtained with other methods such as SIMP [7] or AESO [13]. As expected, the best definition of the topology outline is that produced with the Finer mesh (Figure 4c). Although the features of the design are present in all the mesh models, the Finer mesh allows for better definition of the features of the design. An element size of $1 \%$ of the total length is reasonable for a good definition of the topology outline and it is the one considered in the rest of this work.

\subsection{Gripper mechanism with different target volumes}

The design domain for a gripper mechanism is shown in Figure 5. It is a square of size 200x200mm subdivided using square four node finite elements. A 50x50mm square in the right side is removed from the design domain to allow the mechanism to make its role of gripping the work piece (modelled by the output spring $\mathrm{K}_{\text {out }}$ ).

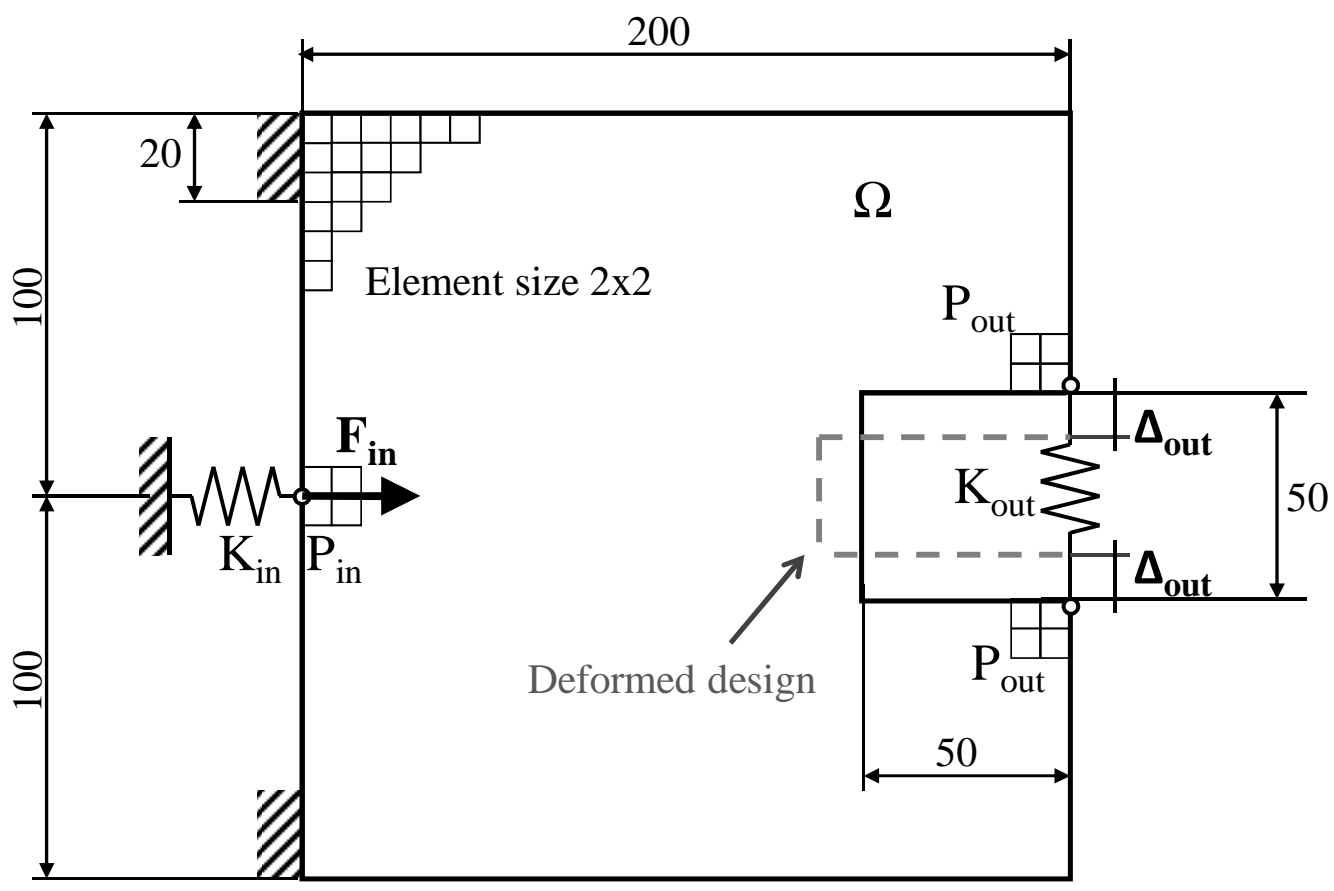

Figure 5. Gripper mechanism (all dimensions are in $\mathrm{mm}$ ) 
In this section, the parameter varied is the target volume while the rest of parameters are maintained equal $\left(\mathrm{F}_{\mathrm{in}}=1 \mathrm{~N}, \mathrm{k}_{\text {out }} / \mathrm{k}_{\mathrm{in}}=1\right.$, a fine mesh, $\left.\mathrm{r}_{\mathrm{min}}=6 \mathrm{~mm}\right)$. The 3 target volumes considered are: 1) $V^{*}=20 \%$; 2) $V^{*}=40 \%$; 3) $V^{*}=60 \%$ of the initial design. Results are shown in Figure 6.

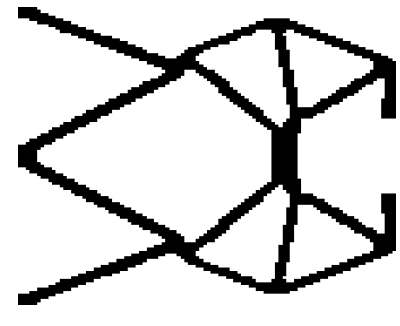

a

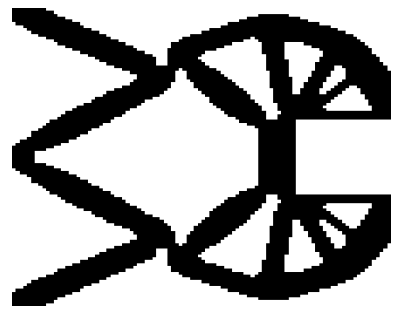

b

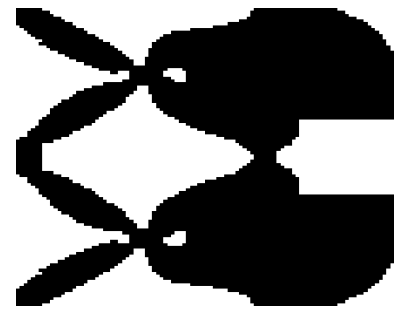

$\mathrm{C}$

Figure 6. Gripper mechanism with a target volume of: a) $20 \%$, b) $40 \%$, c) $60 \%$

It can be observed that material is efficiently distributed to transmit the displacement between the input and output ports, even at low volume fractions (Figure 6a). When the volume fraction increases, the excess of material is distributed where it does not affect the kinematic requirement and the hinges are maintained efficient for the mechanism purpose (Figure $6 \mathrm{~b}$ and Figure 6c).

\subsection{Crunching mechanism with different stiffness ratios}

The design domain for a crunching mechanism is shown in Figure 7. It is a square of size 200x200mm subdivided using square four node finite elements.

In this section, the parameter varied is the stiffness ratio between the input and output points. The formulation used in this work allows the displacement amplification to be controlled by specifying different values of the input and output springs as part of the definition of the problem. A method that obtains topologies for a large range of stiffness ratios allows the designer to represent any possible external conditions of the actuator.

The 3 ratios used are: 1) An elastic work piece with a stiffness ratio of $\mathrm{k}_{\mathrm{out}} / \mathrm{k}_{\mathrm{in}}=0.01 ; 2$ ) an intermediate output stiffness of $\mathrm{k}_{\mathrm{out}} / \mathrm{k}_{\mathrm{in}}=1$ and 3) a stiff workpiece with a stiffness ratio of $\mathrm{k}_{\text {out }} / \mathrm{k}_{\mathrm{in}}=100$. The rest of parameters are maintained equal in the three cases: $\mathrm{F}_{\mathrm{in}}=1 \mathrm{~N}$, $\mathrm{V}^{*}=40 \%$, a fine mesh is used, and $\mathrm{r}_{\min }=6 \mathrm{~mm}$. Results for the three cases defined are shown in Figure 8. 


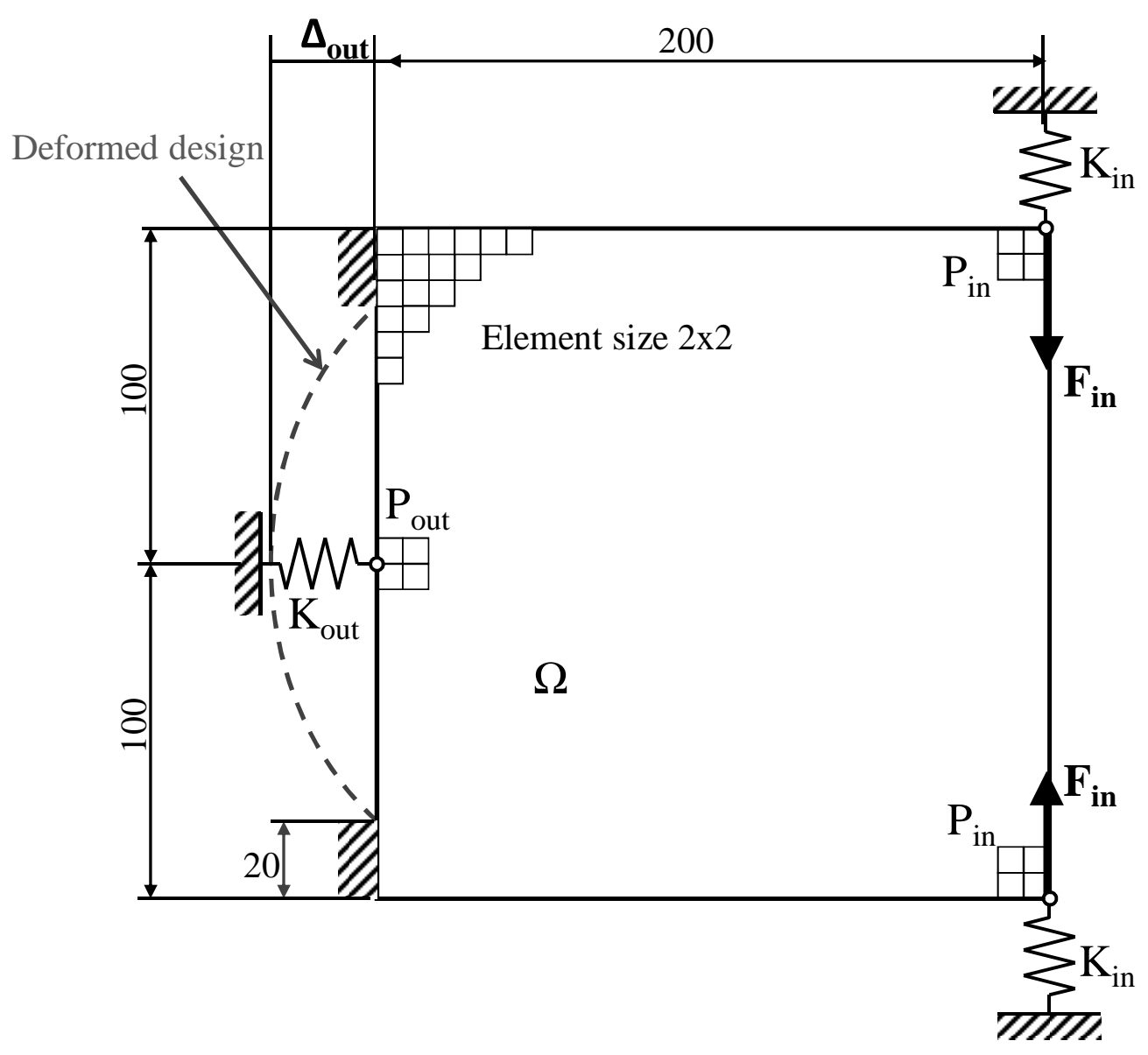

Figure 7. Crunching mechanism (all dimensions are in $\mathrm{mm}$ )

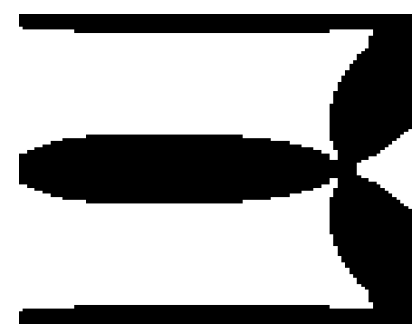

a

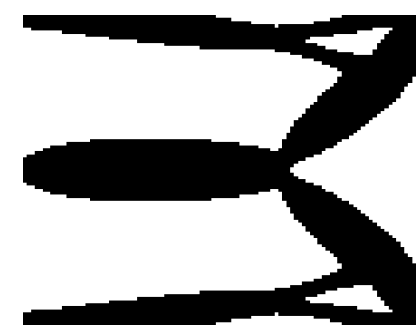

b

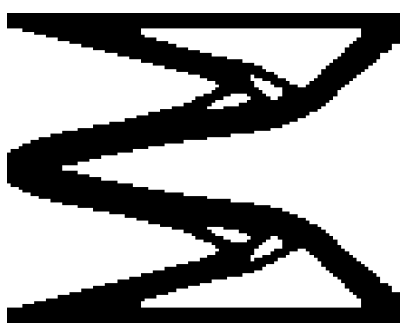

$\mathrm{c}$

Figure 8. Crunching mechanism with a stiffness ratio of: a) kout $/$ kin $=0.01$; b) kout $/$ kin $=1$; and c) kout/kin=100

The obtaining topologies can be interpreted as follows: In general, for a crunching mechanism without any stiffness requirement, the maximum displacement is obtained when the mechanism has vertical bars joining the input and the central bar. That is why when low output stiffness is considered (Figure 8a), the obtained topology has nearly vertical bars between the input point and the central bar. The reaction forces in the output and in the whole mechanism are very small because of the elastic work piece so the mechanisms can "focus" on maximizing the output displacement. On the contrary, if the output stiffness is high (figure $8 c$ ) and, as a consequence, the reaction force in the output is also high, the bars joining the 
input and output points are the most horizontal possible in order to better withstand that reaction force. An intermediate solution between the two extreme cases explained is obtained when the stiffness ratio is the unit (Figure 8b).

\section{CONCLUSIONS}

The Sequential Element Rejection and Admission method presented in this work overcomes the issues noticed in the ESO method used to design compliant mechanisms so far. The main difference of this method with respect to other bi-directional methods that add and remove elements from the design domain is the separate treatment of 'real' and 'virtual' material. Separate criteria for each material model are defined to efficiently add and remove elements and achieve the optimum topology.

The problem of designing compliant mechanisms is defined here as the maximization of the mutual potential energy and a spring model is used to control the input and output stiffness. This formulation meets the flexibility and stiffness requirements necessary to design compliant mechanisms that satisfy the kinematic requirements and at the same time withstand the applied loads.

With the use of three benchmark problems, the proposed method is proven to be robust and versatile for the design of compliant mechanisms by means of topology optimization techniques.

\section{Acknowledgements}

This work was partially supported by the Department of Education of the Government of Navarre with the PhD scholarship of Cristina Alonso Gordoa.

This work was also partially supported by the Ministry of Education and Science in Spain through the project DPI2009-08965.

Their support is greatly appreciated.

\section{REFERENCES}

[1] Sevak, N.M. and Mclaman, C.W. "Optimal synthesis of flexible link mechanisms with large static deflection", ASME, Vol. 83, No. 74-Der, 1974.

[2] Her, I. and Midha, A. "A compliance number concept for compliant mechanisms and type synthesis". Journal of Mechanisms. Transmissions and Auromarion in Design. Transactions of the ASME, Vol. 109(3), pp. 348-355, 1987.

[3] Howell, L.L. “Compliant mechanisms”. New York: John Wiley \& Sons, 2001.

[4] Howell, L. L. and Midha, A. "A generalized loop-closure theory for the analysis and syrithesis of compliant mechanisms", ASME Machine Elemenrs and Machine Dynamics, Vol. 116, pp.491-500, 1994. 
[5] Howell, L. L. and Midha, A. "A method for design of compliant mechanisms with small length flexural pivots” ASME Machine Elemenrs and Machine Dynamics, Vol. 116, pp.280-290, 1994.

[6] Ananthasuresh, G. K., Kota, S. and Gianchandani, Y. "A methodical approach to the design of compliant micromechanisms", Solid State Sensor and Actuator Workshop, pp.189-192, 1994.

[7] Sigmund, O. "On the Design of Compliant Mechanisms Using Topology Optimization", Mech. Struct. \& Mach, Vol. 25:4, pp.493-524, 1997.

[8] Sigmund, O. "Design of multiphysics actuators using topology optimization|part I: Onematerial structures”, Comput. Methods Appl. Engrg., 190, 2001.

[9] Sigmund, O. "Design of multiphysics actuators using topology optimization|part II: Twomaterial structures”, Comput. Methods Appl. Engrg., 190, 2001.

[10]Pedersen, C.B.W., Buhl, T. and Sigmund, O. “ Topology Synthesis of LargeDisplacement Compliant Mechanisms” Int. J. Numer. Meth. Engng., Vol. 50, pp.26832705, 2001.

[11]Parsons, R. and Canfield, S.L. "Developing genetic programming techniques for the design of compliant mechanisms", Struct. Multidisc Optim., Vol. 24, pp.78-86, 2002.

[12] Yulin, M. and Xiaoming, W. "A level set method for structural topology optimization and its applications", Advances in Engineering Software, Vol. 35, pp. 415-441, 2004.

[13] Ansola, R., Vegueria, E., Canales, J. and Tarrago, J.A. "A simple evolutionary topology optimization procedure for compliant mechanism design", Finite Element Analysis and Design, Vol. 44, pp.53-62, 2007.

[14]Lu, K. and Kota, S. "Topology and dimensional synthesis of compliant mechanisms using discrete optimization", ASME, Vol. 128, 2006.

[15] Sharma, D., Deb, K. and Kishore, N.N. "Domain-specific initial population strategy for compliant mechanisms using customized genetic algorithm”, Struct. Multidisc Optim, Vol. 43, pp. 541-554, 2011.

[16]Luo, Z., Tong, L., Luo, J., Wei, P. and Wang, M.Y. "Design of piezoelectric actuators using a multiphase level set method of piecewise constants", Journal of Computational Physics, Vol. 228, pp.2643-2659, 2009.

[17] Saxena, A. "Topology design of large displacement compliant mechanisms with multiple materials and multiple output ports", Struct. Multidisc Optim., Vol. 30, pp.477-490, 2005. 
[18] Saxena, A. "Synthesis of Compliant Mechanisms for Path Generation using Genetic Algoithm”, ASME J. Mech. Des., Vol. 127, pp.745-752, 2005.

[19]Luo, Z. and Tong, L.Y. "A level set method for shape and topology optimization of largedisplacement compliant mechanisms”, Int. J. Numer. Meth. Engng., Vol. 76, pp.862-892, 2008.

[20]Querin, O.M., Steven, G.P. and Xie, Y.M. "Evolutionary Structural optimization using an additive algorithm". Finite Element in Analysis and Design, Vol. 34, p. 18, 2000.

[21] Rozvany, G.I.N. and Querin, O.M. "Theoretical foundations of Sequential Element Rejections and Admissions (SERA) methods and their computational implementations in topology optimization”, Proc. 9th AIAA/ISSMO Symposium on Multidisciplinary Analysis and Optimization, 4-6 September 2002, Atlanta, Georgia, Paper 2002-5521, 2002.

[22] Sigmund, O. and Petersson, J. "Numerical instabilities in topology optimization: A survey on procedures dealing with checkerboards, mesh-dependencies and local minima”, Struct. Optim., Vol.16, pp. 68-75, 1998.

[23] Shield, R.T. and Prager, W. "Optimal Structural Design for Given Deflection”, J. Appl. Math. Phys., Vol. 21, pp.513-523, 1970. 